to explain the periodic ecosystem collapses that have occurred at intervals of roughly 150 million years since Cambrian times (H. Tappan, Palaeogeor., Palaeoclimatol. Palaeoecol., 4, 187; 1968). The analysis by Degens and Stoffers, particularly of deep sediment cores taken from the Black Sea by the Glomar Challenger, will enable such hypotheses to be tested quantitatively and should allow more definite statements to be made about the stresses that accompanied shifts of emphasis in the evolutionary process in the oceans.

These reminders of instability may also contain a word of warning. Natural control mechanisms are fairly delicately balanced and they often work by a process of overshoot and damping rather than by rigorous control within fine limits. As the Baltic countries are finding to their cost, increased oxygen demand resulting from industrial effluent can quickly generate oxygen-deficient bottom water in an enclosed sea. Similar problems have occurred in the Great Lakes of North America and have recently been reported in Lake Baikal. Furthermore, artificial upwellings proposed both as a source of power and as a means for stimulating planktonic growth in the surface waters of the oceans may also generate oxygen-deficient bottom water unless they are located with care.

\title{
Maintenance and evolution of repeated genes in eukaryotes
}

\section{from $D$. Glover}

THE genes for 5S RNA and ribosomal RNA are highly repetitive and occur as tandemly repeated clusters within eukaryotic genomes. These genes are separated by so-called spacer sequences which are not transcribed. Two main theories have been proposed to explain the mechanism by which tandemly arranged sequences are kept relatively homogeneous and how the genes and their spacers have evolved together. At the one extreme, are the sudden correction mechanisms such as Callan's "Master-Slave" hypothesis in which many genes are simultaneously corrected against a master template. At the other extreme, gradual correction mechanisms have been proposed which involve unequal crossing-over between homologous chromatids in such a way that variants could be spread or eliminated from tandem genes. The spacers of the genes for 5S DNA and rDNA in Xenopus laevis have now been subjected to elegant molecular analysis and this has cast some light on these problems.

The 5S DNA of $X$. laevis has an AT-rich spacer which consists largely of repeated units of simple sequence DNA (Brownlee et al., J. molec. Biol., 89, 703; 1974). Caroll and Brown (Cell, 7,$467 ; 1976$ have recently described evidence which indicates that there is considerable heterogeneity with respect to length in this spacer sequence. Cleavage with the restriction endonuclease HindIIT occurs at one site in each 5S gene and generates a series of closely spaced bands each of approximately 700 base pairs. These bands differ in length by regular increments of 14 base pairs which is the length of the simple sequence unit described by Brownlee et al. Carroll and
Brown went on to make partial HindII digests of gradient purified 5S DNA and have cloned these fragments. Each cloned DNA contains up to five of the 5S genes within a DNA plasmid of E. coli (Cell, 7, 477; 1976). Denaturation mapping of these cloned $5 \mathrm{~S}$ genes indicates that adjacent genes can be heterogeneous in length. It is unlikely that such arrangements could be found if sudden correction mechanisms were operating during evolution and Carroll and Brown come down strongly in favour of a mechanism of unequal crossing over between the AT-rich spacers in which the sub-repeats are essentially homologous.

Wellauer et al. (J. molec. Biol., 105, 461 ; 1976) have now made a similar analysis of the ribosomal genes from Xenopus laevis. There are two classes of EcoRI fragments which can be excised from the gene unit. One of these is homogeneous for length whereas the other class of fragment is heterogeneous for length and contains non-transcribed spacer sequences. Four representatives of this latter class have been cloned within $E$. coli and then characterised by homoduplex and heteroduplex mapping. This has revealed two regions within the non-transcribed spacer which vary in length and seem also to consist of repetitious simple sequences. the subunit of which is shorter than 50 base pairs in length. In $X$. laevis the ribosomal genes of oocytes undergo substantial amplification, a process which is thought to occur by a rolling circle mechanism of DNA replication (Hourcade et al., Proc, natn. Acad. Sci. U.S.A., 70, 2926; 1973: Rochaix et al., J. molec. Biol., 87, 473: 1976. If this is so, then one would predict that adjacent rDNA genes from oocytes would be more homogeneous for length than adjacent rDNA genes from somatic tissue. Wellauer et al. ( $J$. molec. Biol., 105, 487; 1976) have examined heteroduplex structures formed between either long singlestrand chromosomal or amplified rDNA and a cloned segment of rDNA and have found that this is indeed the case. In a third paper, the same group has found that not only is there heterogeneity in spacer lengths, but also that the pattern of spacer lengths is significantly different from one animal to another so that it can be followed as a genetic marker (Reeder et al., J. molec. Biol., 105, 507; 1976). The patterns of spacer heterogeneity are stably inherited from one generation to the next and only two animals out of 50 showed changes in their spacer patterns. The mechanism by which these two changes arose remains an open question.

The tandemly repeated gene clusters of Drosophila melanogaster are also producing their share of surprises. The nucleolus organisers of $D$. melanogaster are found on both the $\mathbf{X}$ and the $\mathbf{Y}$ chromosome. In wild-type males there is apparently no meiotic recombination and so the two nucleolus organisers are genetically isolated from one another on non-homologous chromosomes. The rRNA transcribed from either the $\mathrm{X}$ or the $\mathrm{Y}$ seems to be identical however. A single ribosomal gene unit from this organism has been described which from the evidence of DNA reassociation kinetics also contains about 3,000 base pairs of internally repetitious simple sequence. There are two major size classes of rDNA, one in which the repeating gene unit is 17 Kbases in length and the other in which the repeating gene unit is $11-12 \mathrm{~Kb}$ in length (Glover et al., Cell, 5, 149; 1975). Tartof and Dawid (on page 27 of this issue of Nature) have examined the distribution of these two units on the $X$ and $Y$ nucleolus organisers and find that whereas the $11-12 \mathrm{~Kb}$ unit is present on both the $X$ and $Y$ chromosomes, the $17 \mathrm{~Kb}$ unit is found only on the $X$. This suggests that indeed recombination is not occurring between the $X$ and $Y$ rDNAs, unless there is some specific mechanism for eliminating the $17 \mathrm{~Kb}$ units from the $Y$ chromosome.

The mechanism whereby the identity of the rDNA sequences on the $X$ and $Y$ chromosomes is maintained is obscure, and indeed it will be interesting to have a comparison of spacer sequences on the $12 \mathrm{~Kb}$ and $17 \mathrm{~Kb}$ units which have been cloned in $E$. coli. The mechanism of the maintainance and coevolution of these tandomly repeating genes may turn out to be even more complicated than the experiments with Xenopus 5S DNA and rDNA suggest. 